\title{
ONTOGENETIC DEVELOPMENT OF POSTURAL CONTROL IN MAN: ADAPTATION TO ALTERED SUPPORT AND VISUAL CONDITIONS DURING STANCE ${ }^{1}$
}

\author{
HANS FORSSBERG ${ }^{2}$ AND LEWIS M. NASHNER ${ }^{3}$ \\ Neurological Sciences Institute, Good Samaritan Hospital and Medical Center, Portland, Oregon 97209
}

Received August 5, 1981; Revised December 23, 1981; Accepted December 29, 1981

\begin{abstract}
Normal young children ranging in age from $1^{1 / 2}$ to 10 years were assessed in a number of experimental paradigms testing the ability to adapt quickly their strategy of control to altered support surface and visual conditions. The experimental protocols, using a movable platform and visual surround, and the analytic techniques, using EMGs and measures of reaction forces and body motions, were identical to those employed in a complementary study in this issue (Nashner, L. M., F. O. Black, and C. Wall, III (1982) J. Neurosci. 2: 536-544).

The structure of automatic postural adjustments in young children was, with the exception of greater variability, similar to that of adult subjects studied previously. However, young children below the age of $7 \frac{1}{2}$ years were unable to suppress systematically the influence of inputs derived from the support surface or from vision when these provided inappropriate orientation information due to the motion of these surfaces. The discussion emphasizes that the automatic postural adjustments and the context-dependent reweighting of support surface, vestibular, and visual inputs are organizationally separate processes and that the hierarchically lower level automatic process matures before the higher level adaptive processes.
\end{abstract}

The upright equilibrium of the freely standing human is maintained using orientational information derived from three independent sensory sources: somatothesis, vestibular inputs, and vision. Several previous studies of normal adult stance found the most rapid postural reactions (95- to $110-\mathrm{msec}$ latency) to be a class of motor activity distinct from the voluntary system and these reactions therefore were termed "automatic postural adjustments" (Nashner and Cordo, 1981). Furthermore, it was found that automatic postural activities, which provide most of the sway compensation under fixed support surface conditions, are mediated primarily by "support surface" inputs, those derived from the forces and motions of the feet upon the support surface (Nashner, 1977). Following the imposition of brief support surface

\footnotetext{
' This work was supported by National Institutes of Health Grant NS-12661.

${ }^{2}$ Recipient of a visiting fellowship at Good Samaritan Hospital and Medical Center supported by the Swedish Medical Research Council (Grants 19P-5639 and 19F-5896), Norrbacka-Eugeniastiftelsen, and the Neurological Sciences Institute, Good Samaritan Hospital and Medical Center. Permanent address: Department of Physiology III, Karolinska Institutet, S-114 33 Stockholm, Sweden.

${ }^{3}$ To whom correspondence should be addressed at Neurological Sciences Institute, Good Samaritan Hospital and Medical Center, 1120 N. W. 20th Avenue, Portland, OR 97209.
}

displacements, automatic postural adjustments were characterized by relatively fixed temporal and structural patterns of ankle and thigh muscle EMG specified by the waveform of surface displacement (Nashner, 1977; Nashner et al., 1979).

In general, however, the relative weighting among support surface, vestibular, and visual inputs was context dependent. When equilibrium was perturbed by rotations of the support surface with respect to the subject rather than the more usual sway movements of the subject with respect to a fixed support surface, the amplitudes of automatic components of postural activity were attenuated progressively over three to five trials (Nashner, 1976). Following this adaptive change in strategy, appropriate EMG adjustments following sway perturbations were more delayed (175-msec or longer latencies) and now were presumably mediated by vestibular and visual rather than by the now inappropriate support surface inputs. A context-dependent visual modulation of automatic postural adjustments was demonstrated by stabilizing the visual surrounds of the subject unexpectedly during sway perturbations. Under this condition, the amplitudes of automatic EMG adjustments were attenuated and subjects' sway deviations were larger than normal (Nashner and Berthoz, 1978). However, progressive increases in the amplitude of the subsequent five 
EMG adjustments and associated decreases in sway excursions indicated that the weighting of the now inappropriate visual input was being reduced progressively.

Context-dependent reorganization of the weighting of support surface, vestibular, and visual inputs is a complex adaptive process which has not been well defined. In an effort to better understand the characteristics of this adaptive process, normal adults and adult patients with clinically well identified vestibular lesions were exposed to a variety of support surface and visual conditions (Nashner et al., 1982). The most significant finding in this companion study was that vestibular patients, even those whose deficits were subtle by clinical standards, lost their balance whenever motions of support and visual surfaces disturbed the orientation information imparted by these inputs. These patients could maintain balance successfully when deprived of orientation sensations from the support surface and vision, and yet they lost balance when conflicting support surface and visual inputs were introduced. These results supported the hypothesis that rapid adaptation to altered sensory environments is, in fact, a fixed, hierarchical process in which the vestibular system provides the essential inertial-gravitational reference.

This study has explored further the nature of contextdependent weighting of support surface and visual inputs to the posture control system by examining the equilibrium controls of a group of normal children ranging in age from $1 \frac{1 / 2}{2}$ to 10 years. Although the neurophysiological basis for the development of equilibrium control in children is only fragmentary, the available evidence suggests that the vestibular system is operating shortly after birth (Eviatar et al., 1978; Mitchell and Cambon, 1969) but that the ability to adapt to visual conflicts develops much later. Lee and Aronson (1974) reported that infants who recently had learned to stand fell when the closed end of a movable room (with fixed floor) was displaced suddenly. In another study, the postural reactions of seated children to moving visual stimuli were weaker in older (mean age, 16 months) as compared to younger (mean age, 11 months) subjects (Butterworth and Hicks, 1977). Brandt et al. (1976) tested the postural reactions of children exposed to a large rotating visual field and found minimal activity in seated infants (6 to 12 months), maximal reactions in standing 2 - to 5 -year-olds, and decreased effects in 5- to 15-year-olds. These results may well indicate a dominance of visual control in young children. Another plausible explanation is that support surface, vestibular, and visual controls are all equally well developed; however, the young child performing under altered sensory conditions is unable to establish the appropriate context-dependent weighting among the three senses. Hence, young children may demonstrate equally inappropriate responses to conflicting support surface inputs as they did in Lee and Aronson's (1974) study to conflicting vision. If true, this alternate explanation would suggest a developmental process for posture control rather different from that proposed by the above authors. In addition, such a finding would illustrate that the process mediating automatic postural adjustments and the processes which establish the appropriate context-dependent weighting among support surface, vestib- ular, and visual inputs are organizationally separate functions.

In this study, the identical protocols used to evaluate context-dependent reorganization of sensory inputs in patients with vestibular disorders have been applied to a group of 17 young children. The central findings of this study were that: (1) the temporal and spatial structure of automatic adjustments mediated by support surface inputs is similar to that of adults in all, including the youngest, children; (2) young children can maintain their equilibrium when simultaneously deprived of support surface inputs and of vision (eye closure); however, (3) those below 7 to 8 years lost their balance when presented with conflicting visual and support surface inputs. These results suggest that the inability to establish appropriate context-dependent weighting of inputs under altered conditions is a principal cause for the greater instability of young children.

\section{Materials and Methods}

Subject selection. Candidates ranging in age from $1 \frac{1 / 2}{2}$ to 10 years were recruited from several nearby schools and from the families of Good Samaritan Hospital and Medical Center employees. The only criteria for selèction, other than age, were that each child be capable of standing unsupported and that each have no history of neurologic or developmental disorder. Among the 18 male and female children initially recruited, 1 was sufficiently uncooperative to be suspended from tests. The remaining children were divided into four age groups: 4 $1^{1 / 2}$ - to $3^{1 / 2}$-year-olds, all freely standing and walking; 4 $3 \frac{1}{2}$ - to 5 -year-olds; 6 5- to $7 \frac{1}{2}$-year-olds; and $371 / 2$ - to 10 year-olds.

Platform test procedures. All procedures and results of tests on normal adults were described previously (Nashner, 1971, 1976; Nashner and Berthoz, 1978). Their application within the context of this experiment was outlined fully in the companion study (Nashner et al., 1982). The procedures involved the use of an instrumented platform system (Fig. 1 of Nashner et al., 1982) comprised of two movable support surfaces (one for each foot, each independently translates in horizontal and vertical directions and rotates about an axis co-linear with the ankle joint) and a movable visual surface (a 1-m square enclosure open on the back and bottom sides, which rotates about an axis co-linear with the ankle joints). Strain gauges incorporated within each support surface independently measured the torsional forces exerted by the foot. A potentiometer attached to the child's hips via a lightweight rod and belt measured the anteroposterior (AP) sway orientation of the center of gravity with respect to the ankle joints.

The automatic postural system was assessed by quickly displacing both support surfaces forward to backward, causing AP sway in the opposite direction, with the ankle joints being the principal axis of rotation (Nashner, 1977). Orientationally inappropriate support surface inputs were produced by rotating the two support surfaces. The rates of support surface translation were scaled to the height of each child (rates ranged from 15 to 25 $\mathrm{cm} / \mathrm{sec}$ for $250 \mathrm{msec}$ ) to produce a uniform initial rate of AP sway of approximately $20^{\circ} / \mathrm{sec}$. Support surface ro- 
tations were always $20^{\circ} / \mathrm{sec}$ for $250 \mathrm{msec}$ so that the rates of ankle joint rotation were approximately the same during both stimulus conditions.

There were no procedural changes in "support surface" or "visual stabilization" introduced in the vestibular patient study (Nashner et al., 1982). The orientational inputs derived from these surfaces were eliminated by rotating them (axes co-linear with ankle joints) in direct proportion to the measured AP sway motions of the center of body mass.

The techniques used to quantify the temporal and structural organization of automatic EMG adjustments and the sway performance of children were also similar to previous studies. Individual EMGs (pediatric surface electrodes) were processed by full wave rectifying and then bandpass filtering ( 0 to $40 \mathrm{~Hz}$ ) the signal. The amplitudes of automatic postural adjustments were quantified by numerically integrating the processed EMG signals over a 75-msec interval following the onset of activity. As was the case in previous adult studies (Nashner, 1977), the first component of the EMG adjustment in older children occurred repeatedly within a 100 to 175-msec interval. However, as described under "Results," latencies and intervals were much more variable in younger children, especially those within the $3^{1 / 2}$ to $7 \frac{1}{2}$-year-old groups. Therefore, a response was judged to be automatic only when the EMG latency of the appropriate ankle muscle was shorter than $150 \mathrm{msec}$. A maximum 150-msec latency was chosen because, in adults, 150 msec was the absolute earliest that EMG responses occurred in subjects deprived of support surface inputs. Among the 31/2- to $7^{1 / 2}$-year-old group, approximately $20 \%$ of responses occurred at latencies above 150 msec, whereas this value was less than $10 \%$ in other age groups. The organization of EMG patterns then was quantified by specifying the relative latency and amplitude ratios of functionally synergistic ankle and thigh muscles.

The computational techniques used to compare, under different sensory conditions, the stance performance and the ability to adapt of different aged children were both identical to those introduced in the adult vestibular study (Nashner et al., 1982). Because some of the youngest

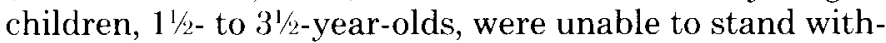
out voluntarily moving about for $50 \mathrm{sec}$, we were forced to reduce test intervals to 10 to $20 \mathrm{sec}$ (appropriate rescaling of performance indices) in a few of these instances.

Protocol. With the few exceptions noted below, the test sequence outlined in Table I (identical to Table II of Nashner et al., 1982) was repeated twice on each child, once during each of two 1-hr sessions. Data from both test sessions were combined in the presentation of results. As already mentioned, we were forced to foreshorten performance tests to 10 to $20 \mathrm{sec}$ for many of the $1 \frac{1}{2}$ - to $31 / 2$-year-olds. In addition, we also found it impossible for children below 5 years to stand with eyes closed for extended intervals of time. Hence, performance tests 2 (normal support surface, eyes closed $\left(\mathrm{S}_{\mathrm{N}} \mathrm{V}_{\mathrm{C}}\right)$ conditions) and 9 (stabilized support surface, eyes closed $\left(\mathrm{S}_{\mathrm{S}} \mathrm{V}_{\mathrm{C}}\right)$ conditions) were not run on children below 5 years.

A final experiment was conducted with several of the
TABLE I

Test sequence for children

\begin{tabular}{|c|c|c|}
\hline Type of Test & Sensory Conditions & $\begin{array}{c}\text { Number and } \\
\text { Duration of } \\
\text { Trials }\end{array}$ \\
\hline 1. Performance & $\begin{array}{l}\text { Normal (fixed) support } \\
\text { and visual surfaces }\end{array}$ & $2,50 \mathrm{sec}$ \\
\hline 2. Performance & $\begin{array}{l}\text { Normal support surface, } \\
\text { eyes closed }\end{array}$ & $2,50 \mathrm{sec}$ \\
\hline 3. Performance & $\begin{array}{l}\text { Normal support surface, } \\
\text { stabilized vision }\end{array}$ & $2,50 \mathrm{sec}$ \\
\hline $\begin{array}{l}\text { 4. Transient support } \\
\text { surface translations }\end{array}$ & $\begin{array}{l}\text { Normal support and } \\
\text { visual conditions }\end{array}$ & $\begin{array}{l}5 \text { forward, } 1 \\
\text { sec }\end{array}$ \\
\hline $\begin{array}{l}\text { 5. 'Transient support } \\
\text { surface rotations }\end{array}$ & $\begin{array}{l}\text { Normal support and } \\
\text { visual conditions }\end{array}$ & $\begin{array}{l}5 \text { "toes up," } \\
1 \mathrm{sec}\end{array}$ \\
\hline $\begin{array}{l}\text { 6. Transient support } \\
\text { surface translations }\end{array}$ & $\begin{array}{l}\text { Normal support and } \\
\text { visual conditions }\end{array}$ & $\begin{array}{c}5 \text { backward, } \\
1 \mathrm{sec}\end{array}$ \\
\hline $\begin{array}{l}\text { 7. Transient support } \\
\text { surface rotations }\end{array}$ & $\begin{array}{l}\text { Normal support and } \\
\text { visual conditions }\end{array}$ & $\begin{array}{l}5 \text { "toes } \\
\text { down," } 1 \\
\text { sec }\end{array}$ \\
\hline 8. Performance & $\begin{array}{l}\text { Stabilized support } \\
\text { surface, normal vision }\end{array}$ & $2,50 \mathrm{sec}$ \\
\hline 9. Performance & $\begin{array}{l}\text { Stabilized support } \\
\text { surface, eyes closed }\end{array}$ & $2,50 \mathrm{sec}$ \\
\hline 10. Performance & $\begin{array}{l}\text { Stabilized support and } \\
\text { visual surfaces }\end{array}$ & $2,50 \mathrm{sec}$ \\
\hline $\begin{array}{l}\text { 11. Transient support } \\
\text { surface translations }\end{array}$ & $\begin{array}{l}\text { Stabilized support } \\
\text { surface, eyes closed }\end{array}$ & $\begin{array}{l}5 \text { forward, } 1 \\
\text { sec }\end{array}$ \\
\hline $\begin{array}{l}\text { 12. Transient support } \\
\text { surface translations }\end{array}$ & $\begin{array}{l}\text { Stabilized support } \\
\text { surface, eyes closed }\end{array}$ & $\begin{array}{l}5 \text { backward, } \\
1 \text { sec }\end{array}$ \\
\hline
\end{tabular}

youngest children in order to examine the coordination of postural adjustments during arm movements (in adult studies, Cordo and Nashner, 1982). Each child grasped a handle (strain gauges measured handle force), holding it in front of the chest in the right hand with elbow semiflexed. Only one of the two arm movement paradigms used in the adult study, unexpected displacements of the handle, was repeated with these young children, as they did not react consistently to commands to pull upon the handle. During handle displacements, EMGs of the biceps muscle of the right arm and handle force were recorded along with the EMG activity of ankle muscles.

\section{Results}

Automatic postural adjustments. A common misconception is that small children have an easier task maintaining equilibrium than adults, since their center of gravity is "closer to the ground." The truth is the opposite and can easily be tested by balancing a short and long stick, respectively, on the fingertips. The shorter stick has the faster rate of increase of sway and therefore requires more rapid correction following a perturbation.

The latencies of automatic adjustments of young children were more variable than those of adults (Fig. 1). Greater temporal variability, coupled with the fact that the responses of young children were never faster than those of adults, meant that the responses of children below $7 \frac{1}{2}$ years of age were, on the average, significantly slower than those of adults. The consequence of more erratic and, on the average, slower EMG adjustments can be seen in the records of a typical 2-year-old during forward (Fig. 2A) and backward (Fig. 2B) displacements. To illustrate the greater temporal and amplitude varia- 


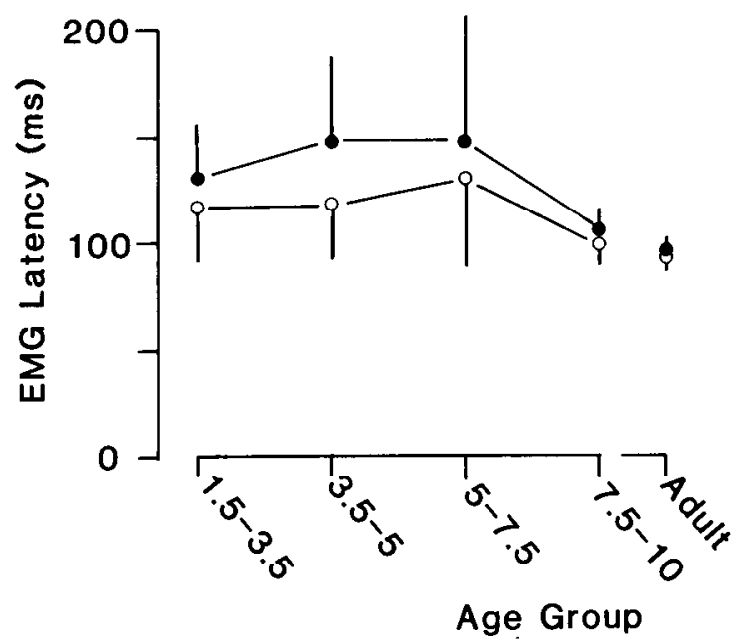

Figure 1. Average ( $\pm \mathrm{SD})$ EMG latencies of responses to forward AP sway perturbations (gastrocnemius, $)$ ) and to backward sway perturbations (tibialis anterior, $\bigcirc$ ). Adult data were derived from Nashner, 1977.

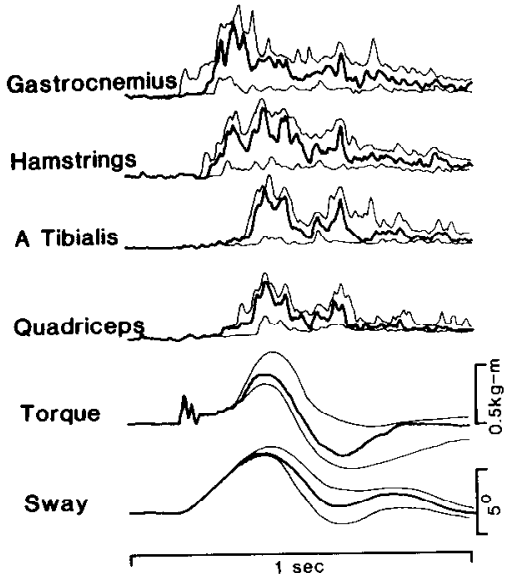

A

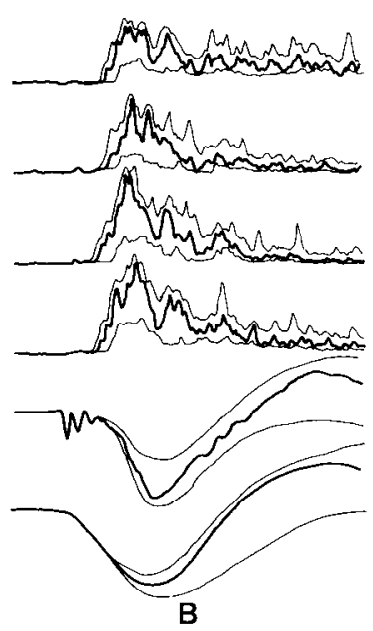

B
Figure 2. Five-trial ensemble averages (heavy lines) and upper and lower bounds (fine lines) of the EMG, torque, and sway responses of a 2-year-old to forward perturbations $(A)$ and backward perturbations $(B)$.

bility of EMG adjustments of the youngest children compared to older children and adults, both the ensemble averages (heavy lines) and the upper and lower bounds of responses (fine lines) are included in this figure. During both forward and backward displacements, slower EMG responses and faster rates of sway acceleration meant that the resulting sway excursions of the 2-yearold were larger and tended to be more oscillatory than those of older children (see Fig. 3) and of adults (see Fig. 3 of Nashner et al., 1982).

Despite the age-dependent differences in the dynamics of AP sway responses, there was a good deal of similarity in the temporal and spatial structure of automatic postural adjustments among all children and adults tested. The gastrocnemius and its proximal synergist, hamstrings, were activated sequentially during forward AP sway. What was not observed in older children and adults was the activation of the anterior tibialis-quadriceps synergy approximately $150 \mathrm{msec}$ following that of gastrocnemius-hamstrings. However, this secondary response was approximately correlated with the onset of backward motion during the resulting sway oscillation. Therefore, this component may, in fact, not represent a structural difference but merely the fact that small children sway at higher frequencies. The co-activation of all four postural muscles during backward sway displacements was a structural pattern not seen regularly in older children and adults. Although the possible significance of antagonist co-activation was not appreciated at the time, a tendency toward increased co-activation also was observed during the most rapid backward displacements of adults in a previous study which quantified the relations between displacement rates and EMG responses over a wide range of stimulus velocities (Nashner and Cordo, 1981). Hence, co-activation as well may be a structural difference or again may be due to the greater instability of the child short in stature.

Similarities between adults and somewhat older children are illustrated by the ensemble averages (heavy lines) and the upper and lower bounds (fine lines) of EMG, torque, and sway responses of a 6-year, 7-monthold to five consecutive forward and backward displacements (Fig. 3, $A$ and $B$, respectively). Activation of hamstrings followed that of gastrocnemius by approximately $20 \mathrm{msec}$ and the two were activated in equal proportion. Activation of the quadriceps was in equal proportion to that of the anterior tibialis and approximately 10 msec later during backward sway displacements. Little if any oscillation or co-activation of antagonists was noted in this child.

The principal findings illustrated by the examples in Figures 2 and 3 are summarized for all children in Table II. For each age group, the temporal (relative latency of distal and proximal synergists) and the spatial (amplitude ratios between synergists and distal antagonists) are averaged among all children in each age group. With the single exception of greater co-activation of antagonists during backward sway (gastrocnemius/tibialis ratio) of
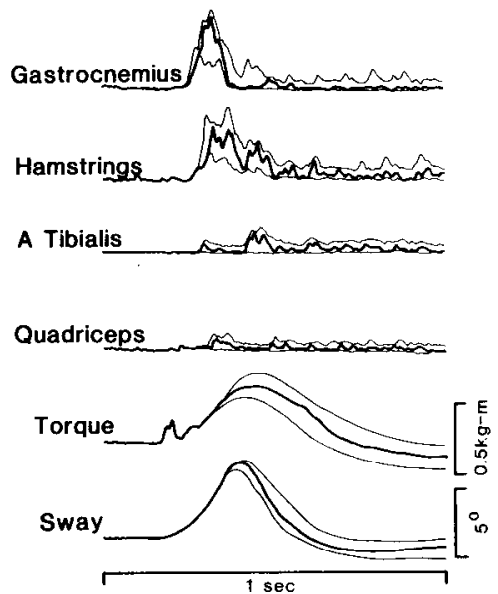

A
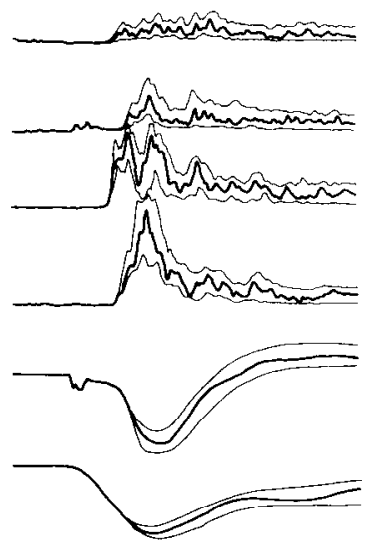

B

Figure 3. Five-trial ensemble averages (heavy lines) and upper and lower bounds (fine lines) of the EMG, torque, and sway responses of a 6-year, 7-month-old child to forward perturbations $(A)$ and backward perturbations $(B)$. 
TABLE II

Structure of automatic postural adjustments in children

\begin{tabular}{lccccccc}
\hline \multirow{2}{*}{$\begin{array}{c}\text { Age } \\
\text { Group }\end{array}$} & \multicolumn{2}{c}{ Forward Sway Adjustments } & & \multicolumn{3}{c}{ Backward Sway Adjustments } \\
\cline { 2 - 3 } \cline { 6 - 8 } & $\begin{array}{c}\text { G to } \mathrm{H} \\
\text { Latency }^{a}\end{array}$ & $\begin{array}{c}\mathrm{H} / \mathrm{G} \\
\text { Ratio }^{b}\end{array}$ & $\begin{array}{c}\text { T/G } \\
\text { Ratio }^{c}\end{array}$ & & $\begin{array}{c}\text { T to Q } \\
\text { Latency }\end{array}$ & $\begin{array}{c}\text { Q/T } \\
\text { Ratio }\end{array}$ & $\begin{array}{c}\text { G/T } \\
\text { Ratio }^{c}\end{array}$ \\
\hline $1^{1 / 2-31 / 2}$ & $20 \pm 25$ & \pm 0.40 & $0.16 \pm 0.05$ & & $41 \pm 36$ & \pm 0.38 & $0.88 \pm 0.40$ \\
$3^{1 / 2-5}$ & $34 \pm 21$ & \pm 0.52 & $0.18 \pm 0.07$ & & $23 \pm 28$ & \pm 0.40 & $0.65 \pm 0.20$ \\
$5-7^{1 / 2}$ & $20 \pm 25$ & \pm 0.35 & $0.15 \pm 0.09$ & & $25 \pm 37$ & \pm 0.44 & $0.45 \pm 0.25$ \\
$7^{1 / 2-10}$ & $10 \pm 12$ & \pm 0.30 & $0.10 \pm 0.04$ & & $13 \pm 20$ & \pm 0.28 & $0.22 \pm 0.10$ \\
Adults $^{d}$ & $22 \pm 16$ & \pm 0.16 & $0.13 \pm 0.05$ & & $8 \pm 7$ & \pm 0.28 & $0.18 \pm 0.10$ \\
\hline
\end{tabular}

${ }^{a}$ Mean delay $( \pm \mathrm{SD})$ of proximal activation following distal muscle activation. The abbreviations used are: $G$, gastrocnemius; $H$, hamstrings; $Q$, quadriceps; $T$, tibialis.

${ }^{b}$ FMG gains for each child were normalized for unit ratios. The standard deviations of the amplitude ratios for all children in each group are given.

- $\mathrm{T} / \mathrm{G}$ and $\mathrm{G} / \mathrm{T}$ ratios quantify the level of co-activation of distal antagonists during forward and backward sway adjustments, respectively. Ratio values approaching unity indicate greater co-activation.

${ }^{d}$ Adult data were derived from Nashner, 1977.

1/2- to 5 -year-old groups, the principal structural features of automatic adjustments were the same for all groups.

Further insight into the organization of the automatic postural system of the standing young child was gained by observing the postural adjustments associated with unexpected displacements of a handle which the child grasped while freely standing. In adult subjects, postural adjustments associated with arm pulls began prior to those elaborating the actual arm movement and were temporally and structurally similar to those stabilizing support surface-induced sway (Cordo and Nashner, 1982). In 4 children tested, including the youngest who would cooperate ( 1 year, 9 months old), activation of the stabilizing gastrocnemius occurred prior to that of the biceps which was directly stretched by the arm pull (Fig. 4). Thus, a basic coordination pattern responsible for maintaining stance equilibrium during voluntary arm movements against an external load was also relatively intact in young children.

Performance with normal support surface and altered visual inputs. When provided a fixed support surface, all children performed well below the limits of stability despite alterations in visual conditions. Figure 5 illustrates the average $( \pm \mathrm{SD})$ performance ratios of each age group with eyes open $\left(\mathrm{S}_{\mathrm{N}} \mathrm{V}_{\mathrm{N}}\right)$, eyes closed $\left(\mathrm{S}_{\mathrm{N}} \mathrm{V}_{\mathrm{C}}\right)$, and stabilized visual $\left(\mathrm{S}_{\mathrm{N}} \mathrm{V}_{\mathrm{S}}\right)$ conditions. While differences in the three performance ratios for each age group were, in nearly all instances, not significantly different, there was a trend toward greater stability with greater visual input and a significant improvement in stability with increased age. However, $7 \frac{112}{2}$ - to 10 -year-olds still swayed nearer to their limits of stability than did adults. (Normal adult data were derived from Nashner et al., 1982.) The above results are consistent with the preceding observations that automatic EMG adjustments are relatively well developed but more erratic in temporal and spatial structure in young children. Apparently, eye closure or unexpected stabilization of the visual surrounds alone does not greatly interfere with the child's performance as long as support surface inputs provide useful orientation information.

Performance with alterations in both support surface and visual conditions. All children maintained their balance when useful support surface inputs were eliminated by stabilizing the support surface with respect to the AP sway orientation of the center of body mass $\left(\mathrm{S}_{\mathrm{S}} \mathrm{V}_{\mathrm{N}}\right.$; Fig. 5). Subsequent eye closure of children aged 5 and above did little to further destabilize posture $\left(\mathrm{S}_{\mathrm{S}} \mathrm{V}_{\mathrm{C}}\right.$ conditions). However, when subsequently reopening their eyes within a stabilized visual surround $\left(\mathrm{S}_{\mathrm{S}} \mathrm{V}_{\mathrm{S}}\right.$ conditions), all but 1 child below $6^{1 / 2}$ years lost balance. This result is dramatized by the average performance ratios of 1.0 for all children aged 5 years or less in the $\mathrm{S}_{\mathrm{S}} \mathrm{V}_{\mathrm{S}}$ condition, and ratio values near unity in the 5- to $7 \frac{1}{2}$ year-old group under this condition. In contrast, children above $7 \frac{1}{2}$ years and adults were approximately as stable with their eyes closed and with vision stabilized, in both instances remaining well below their limits of stability.

Figure 6 illustrates representative AP sway and EMG signals of 3 children performing under normal support surface conditions with eyes open $(A)$, stabilized support surface conditions with eyes open $(B)$, stabilized surface conditions with eyes closed $(C)$, and finally, stabilized support surface and visual conditions $(D)$. When the 1-

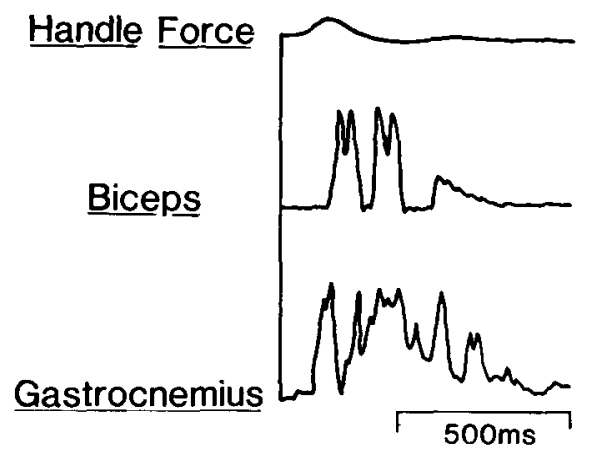

\section{1 yr $\underline{9 m o}$}

Figure 4. Representative EMG and force responses of a 1 year, 9-month-old child to the unexpected pull of a hand-held manipulandum.

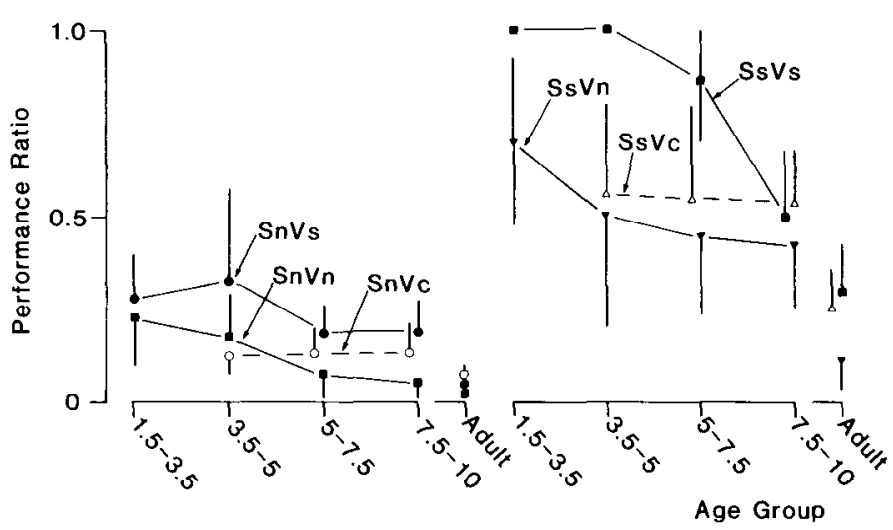

Figure 5. Average ( $\pm \mathrm{SD}$ ) performance ratios of different age groups of children standing under six different conditions: on the left side with fixed support surface and eyes open $\left(S_{N} V_{N}\right)$, eyes closed $\left(S_{N} V_{C}\right)$, and the visual surface stabilized $\left(S_{N} V_{S}\right)$; on the right side with support surface stabilized and eyes open $\left(S_{S} V_{N}\right)$, eyes closed $\left(S_{S} V_{C}\right)$, and the visual surface also stabilized $\left(S_{S} V_{S}\right)$. 


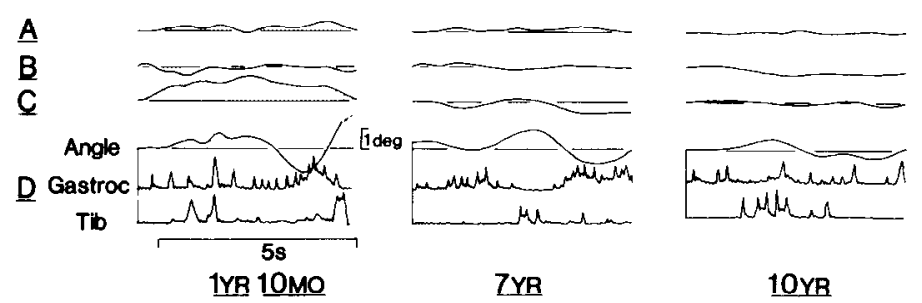

Figure 6. Representative examples of AP sway motions of children standing with eyes open on a fixed support surface $(A)$, with eyes open on a stabilized support surface $(B)$, with eyes closed on a stabilized support surface $(C)$, and with both support and visual surfaces stabilized $(D)$. The records in $D$ also include ankle muscle EMGs. The dotted portions indicate lost balance. The abbreviations used are: Gastroc, gastrocnemius; Tib, tibialis.

year, 10-month-old performed with both surfaces stabilized $\left(\mathrm{S}_{\mathrm{S}} \mathrm{V}_{\mathrm{S}}\right.$ conditions, $\left.D\right)$, there was a long delay from the onset of spontaneous forward sway (downward on $A P$ record) to activation of the appropriate gastrocnemius muscle. Subsequently, the child began to sway backward, this time initiating the appropriate anterior tibialis response only after balance had been lost (dolled portion of $A P$ record). In contrast, the appropriate AP sway modulations of EMG activity were accomplished much more quickly in the 7-and 10-year-olds, and sway oscillations remained within stable limits.

Adaptation to altered support surface conditions. The interpretation that children below the critical age of $7^{1 / 2}$ years cannot suppress the influence of sensory inputs providing inappropriate orientation information was tested further by exposing children to unexpected "toes up" and "toes down" rotational displacements of the support surface. Under this unusual stimulus condition, changes in the rotational positions of the ankle joints followed a similar trajectory as during AP sway perturbations, except now the ankle inputs were uncorrelated with sway. In normal adult subjects, inappropriate, automatic adjustments were attenuated progressively during three to five consecutive rotational perturbations. The ability of children of different ages to attenuate the influence of orientationally inappropriate support surface inputs was characterized by an adaptation ratio, which would be 0 or nearly so whenever the amplitudes of the fourth and fifth EMG responses to surface rotation were much smaller than those of the first and second.

Figure 7 illustrates the average $( \pm \mathrm{SD})$ adaptation ratios for the different age groups between $1 \frac{1 / 2}{2}$ and 10 years. The ratios computed for "toes up" (gastrocnemius) and toes down (anterior tibialis activation) have been combined within each age group. In the two youngest age groups, the amplitude of inappropriate adjustments were highly variable from one trial to the next; however, amplitude changes were not related systematically to the sensory conditions. Because the latencies as well as the amplitudes of activation were highly variable in 5 - to $71 / 2$ year-old children, we found it impossible to distinguish consistently the automatic component of response (see "Materials and Methods") and therefore did not calculate adaptation ratios for this group. In contrast, children above $7 \frac{1}{2}$ years consistently attenuated EMG amplitudes following exposure to a rotating support surface. The

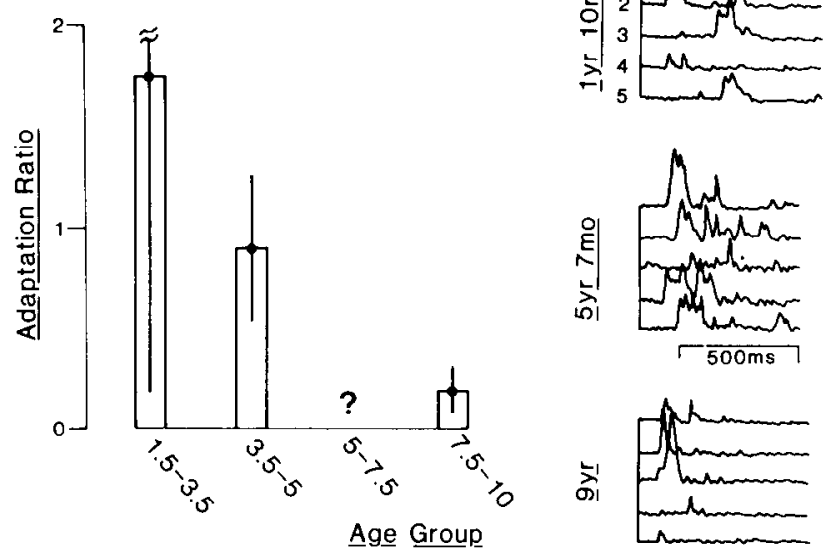

Figure 7. Average $( \pm \mathrm{SD})$ adaptation ratios for each age group computed during sequences of five "toes up" and "toes down" support surface rotations. Insets at right show representative sequences of five consecutive gastrocnemius responses to "toes up" rotations.

inset on the right side of Figure 7 shows representative EMG records for five "toes up" support surface rotations in 3 children. Note the large variations in latency and amplitude of the 5-year, 7-month-old. Although these particular records could not be used to calculate the adaptation ratio for the reason stated above, they nevertheless suggest an inability to suppress inappropriate automatic postural adjustments.

\section{Discussion}

The postural controls of young children were distinguished from those of older children and adults in several aspects. Under normal sensory conditions, the shorter stature of children and the greater variability of their automatic postural adjustments combined to produce sway responses larger, more variable, and more oscillatory than those of adults. When deprived of support surface inputs, visual inputs, or both, all young children performed within stable limits but, again, with larger and more variable oscillations than adults. Under conflicting sensory conditions, younger children, like the adult patients with subtle vestibular deficits (Nashner et al., 1982) often responded inappropriately and lost their balance. In contrast, older children and normal adults performed equally well under the deprived and the conflicting sensory conditions.

Automatic postural controls of young children. The larger and more oscillatory sway response of the young child performing under normal sensory conditions cannot, by itself, be interpreted as developmental immaturity of the automatic postural control system. Since the latency of adjustment must decrease in proportion to the increase in the rate of sway acceleration to maintain the same level of performance, a 70-cm-tall 2-year-old would have to respond at approximately $65 \mathrm{msec}$ to equal the performance of a $180-\mathrm{cm}$-tall adult responding at 100 msec. Physiological evidence suggests, however, that the latency of automatic postural adjustments in young children should be approximately equal that of adults. While the velocity of nerve conduction is lower in infants and increases especially during the first years of life 
(Gamstorp, 1963), the distance to and from the spinal cord is also shorter. The increases in fiber diameter and of intermodal length during development roughly compensate for the elongation of the peripheral nerves, thereby keeping the conduction time roughly constant (Berthold, 1968).

The directional specificity, the distal-to-proximal sequencing of synergists, and the coordination of leg and arm muscles (Table II; Fig. 4) reflect that rather complex control networks controlling an array of postural muscles are developed in the youngest children able to stand and walk without support. Several motor functions (for example, locomotion, respiration, and mastication) are controlled by central networks at lower levels of the motor hierarchy and are characterized by automatized and stereotyped behaviors (Wilson, 1961; Grillner, 1975; Bradley et al., 1975; Dellow and Lund, 1971). The transformation of these stepping movements to an efficient plantigrade gait and the capability to adapt the step cycle emerge only after the 2nd year, when higher level pathways begin to mature (Forssberg and Wallberg, 1980; H. Forssberg, unpublished observations). In infants, stepping movements, presumably generated by the central pattern generators of the spinal cord, can be produced directly after birth (Peiper, 1961). However, to adapt these stereotyped activities to the environment or to coordinate their actions with those of other motor functions, the influence of higher centers is needed (Sears, 1971; Forssberg et al., 1980; von Euler, 1981). In a similar way, we interpret the appearance of stereotyped, automatic postural adjustments in children just able to stand and walk freely as central networks which are inherent and organized within lower levels of the motor hierarchy.

Unlike the system for the automatic control of posture and equilibrium, those responsible for reorganizing sensory inputs to posture within altered sensory environments were relatively undeveloped in children below $7 \frac{1}{2}$ years. Below this critical age, variations in the amplitudes of automatic adjustments were as large as those observed in adults performing within a variety of different contexts; however, in younger children, amplitude variations appeared random rather than related to the environmental context. Younger children performed under deprived sensory conditions, suggesting a significant level of development of vestibular posture controls; yet, they lost balance or became highly unstable under conflicting sensory conditions. Although we interpreted this as the strongest evidence for lack of development of a hierarchically higher level process, we also viewed variability as further evidence that adaptive mechanisms are undeveloped in young children. The trial-to-trial variations in EMG adjustments suggest that young children may randomly, rather than systematically, change the weighting of support surface, vestibular, and visual inputs. While automatic postural adjustments provided the bulk of stabilization during some trials, automatic responses were weaker and/or more delayed and therefore less influential in others. Perhaps the random reweighting of sensory inputs to posture, especially prominent in the years just prior to development of adult adaptive capabilities, is a necessary element of the process for developing adaptive capabilities.

Within the limitations of our experimental paradigm at least, the performance of children below the critical age of $7 \frac{1}{2}$ years resembled that of vestibular deficit patients (Nashner et al., 1982). The automatic postural adjustments of both groups were organized normally, although neither vestibular patients or young children appropriately suppressed the influence of conflicting support surface and/or visual inputs. Based upon this evidence alone, however, we cannot attribute this developmental immaturity exclusively to either the sensory apparatus of the vestibular system or to higher centers of the CNS. The scanty evidence already cited in the introduction suggests that the vestibular receptors and primitive vestibular reflexes are both intact in infants. This is supported by a finding that 5 - to $7^{1 / 2}$-year-olds could stand while concurrently deprived of useful vision and support surface inputs (eye closure and stabilized support), a task which could not be performed by patients without vestibular inputs (Nashner et al., 1982). However, we also found in the adult vestibular study that subtly altered vestibular function impaired the ability to adapt to conflicts but not to perform under deprived sensory conditions. Hence, immaturity may be either in the receptor apparatus, within higher CNS centers, or in both of these processes.

The emergence of the organizationally simplest behaviors during development prior to those for performing the more complex "integrative" functions has been a common finding within the human and the animal developmental literature. Hence, the knowledge that the automatic components of postural activity are relatively well developed in young children while adaptive processes which modify them to suit the environmental conditions of the task are not strengthens the viewpoint that these are two organizationally distinct processes within lower and higher levels, respectively, of a hierarchically structured system. Our current view is that the automatic postural system is an elementary unit of motor action that can be integrated into any number of different complex motor behaviors and environmental contexts. The performance limitations of the young child seem to result in part at least from his inability to coordinate systematically these elementary units of action within the broader context of a complex motor act. However, in the case of posture control, the child's performance also is hampered by a shorter stature which requires more rapid and continuous correction of sway motions.

\section{References}

Berthold, C. H. (1968) Ultrastructure of postnatally developing peripheral nodes of Ranvier. Acta Soc. Med. Upsal. 73: 145-168.

Bradley, G. W., C. von Euler, I. Marttila, and B. Roos (1975) A model of central and reflex inhibition of inspiration in the cat. Biol. Cybern. 19: 105-116.

Brandt, Th., D. Wenzel, and J. Dichgan (1976) Die entwicklung der visuellen Stabilisation des aufrechten Standes beim Kind: ein Reifezeichen in der Kinderneurologie. Arch. Psychiat. Nervenkr. 223: 1-13.

Butterworth, G., and L. Hicks (1977) Visual proprioceptive and postural stability in infancy. A developmental study. Perception 6: 255-262.

Cordo, P. J., and L. M. Nashner (1982) Properties of postural adjustments associated with rapid arm movements. J. Neurophysiol. 47: 287-302. 
Dellow, P. G., and J. P. Lund (1971) Evidence for central timing of rhythmical mastication. J. Physiol. (Lond.) 215: 1-13.

Eviatar, L., and A. Eviatar (1978) Neurovestibular examination of infants and children. Adv. Otorhinolaryngol. 23: 169-191.

Forssberg, H., and H. Wallberg (1980) Infant locomotion: A preliminary movement and electromyographic study. In Children and Exercise IX, K. Berg and B. Eriksson, eds., pp. 32-40, University Park Press, Baltimore.

Forssberg, H., S. Grillner, and J. Halbertsma (1980) The locomotion of the low spinal cat. I: Coordination within a hindlimb. Acta Physiol. Scand. 108: 269-281.

Gamstorp, I. (1963) Normal conduction velocity of ulnar, median and peroneal nerves in infancy, childhood and adolescence. Acta Paediatr. Scand. Suppl. 146: 68-76.

Grillner, S. (1975) Locomotion in vertebrates: Central mechanisms and reflex interaction. Physiol. Rev. 55: 247-304.

Lee, D., and E. Aronson (1974) Visual proprioceptive control of standing in human infants. Percept. Psychophys. 15: 529-532.

Mitchell, T., and K. Cambon (1969) Vestibular response in the neonate and infant. Arch. Otolaryngol. 90: 556-557.

Nashner, L. M. (1971) A model describing the vestibular detertion of body sway motion. Acta Otolaryngol. (Stockh.) 72 429-436.

Nashner, L. M. (1976) Adopting reflexes controlling the human posture. Exp. Brain Res. 26: 59-72.
Nashner, L. M. (1977) Fixed patterns of rapid postural responses among leg muscles during stance. Exp. Brain Res. 30: 13-24.

Nashner, L. M., and A. Berthoz (1978) Visual contribution to rapid motor responses during posture control. Brain Res. 150: 403-407.

Nashner, L. M. and P. J. Cordo (1981) Relation of postural responses and reaction-time voluntary movements in human leg muscles. Exp. Brain Res. 43: 395-405.

Nashner, L. M., M. Woollacott, and G. Tuma (1979) Organization of rapid response to postural and locomotor-like perturbations of standing man. Exp. Brain Res. 36: 463-476.

Nashner, L. M., F. O. Black, and C. Wall, III (1982) Adaptation to altered support and visual conditions during stance: $\mathrm{Pa}$ tients with vestibular deficits. J. Neurosci. 2: 536-544.

Peiper, A. (1961) Cerebral Function in Infancy and Childhood, Consultants Bureau, New York.

Sears, T. A. (1971) Breathing: A sensory-motor act. Sci. Basis Med. Annu. Rev. 48: 129-147.

von Euler, C. (1981) Some aspects on speech breathing physiolngy. In The Motor Control of Speech, S. Grillner, A. Person, B. Lindblom, and J. Lubker, eds., Pergamon Press, Elmsford, $\mathrm{NY}$, in press.

Wilson, D. M. (1961) The central nervous control of flight in a locust. J. Exp. Biol. 38: 471-490. 